\title{
Cyclin A is a reliable proliferation marker in endometrial cancer cell lines
}

\author{
KAYO HORIE $^{1}$, HAYATE YAMAMOTO ${ }^{2}$, KOUHEI KARUBE ${ }^{2}$, KAI TAKEBAYASHI ${ }^{1}$, \\ HIRONORI YOSHINO $^{1}$, HARUHIKO YOSHIOKA ${ }^{1}$ and JUN WATANABE ${ }^{1}$ \\ ${ }^{1}$ Department of Bioscience and Laboratory Medicine, Hirosaki University Graduate School of Health Sciences; \\ ${ }^{2}$ Department of Medical Technology, Hirosaki University School of Health Sciences, Hirosaki, Aomori 036-8564, Japan
}

Received November 21, 2018; Accepted February 28, 2019

DOI: $10.3892 / \mathrm{ol} .2019 .10135$

\begin{abstract}
Cyclin A, a cell cycle regulatory protein, promotes cell proliferation and has been observed to be highly expressed in cancer and to promote tumor growth; however, its value as a marker for endometrial carcinoma has not yet been established. Accordingly, the aim of the present study was to clarify whether cyclin A can be used as a cell proliferation marker using the endometrial carcinoma cell lines Ishikawa and HEC-50B, derived from patients with low-grade and high-grade cancer, respectively. The expression of cyclin A was determined by flow cytometry using double staining with FITC and 7-AAD, and immunocytochemical staining. The results were compared to those of $\mathrm{Ki}-67$, the widely used cell proliferation marker that is considered to be a prognostic marker in endometrial cancer. The flow cytometry results revealed that cyclin A expression was significantly higher in HEC-50B than in Ishikawa cells during the logarithmic growth phase. In addition, cyclin A expression was consistently higher than $\mathrm{Ki}-67$ expression in the examined cell lines. Immunocytochemical staining confirmed cyclin A expression in HEC-50B and Ishikawa cells, demonstrating significantly higher expression during the logarithmic growth phase than during the stationary phase. By contrast, Ki-67 was expressed in almost $90 \%$ of the cells, irrespective of their growth state. These results indicate that cyclin A expression is significantly increased in cells with higher proliferative ability and is specifically expressed in cells that have passed the G1-S checkpoint. Therefore, cyclin A may be a reliable proliferation biomarker for endometrioid carcinoma.
\end{abstract}

Correspondence to: Dr Kayo Horie, Department of Bioscience and Laboratory Medicine, Hirosaki University Graduate School of Health Sciences, 66-1 Hon-cho, Hirosaki, Aomori 036-8564, Japan

E-mail: k-horie@hirosaki-u.ac.jp

Key words: endometrial cancer, cyclin A, flow cytometry, immunocytochemical staining, cell-proliferation marker

\section{Introduction}

Endometrial carcinoma is the most common gynecologic malignancy worldwide; moreover, in Japan, there has been a continual annual increase in its incidence, which is associated with lifestyle changes (1). Diagnosis at an early stage leads to high survival rates, whereas patients diagnosed with advanced-stage or recurrent disease have a poor prognosis (2). Thus, considerable research attention has focused on identifying specific markers for early-stage endometrial carcinoma (3).

$\mathrm{Ki}-67$ is a widely used proliferation marker and prognostic factor in cancer (4), since Ki-67 antibodies recognize a nuclear protein that is expressed only in proliferating cells (5). Moreover, the Ki-67-positive population is detected mainly among proliferating cells in diverse cell types during the active phases of the cell cycle ( $11, \mathrm{~S}, \mathrm{G} 2$, and M phases), and is absent among cells in the resting/quiescent phase (G0 phase) $(5,6)$. Ki-67 expression is strongly associated with cell proliferation and tumor growth and is thus widely used in routine pathological investigations as a proliferation marker; moreover, Ki-67 is well characterized at the molecular level and is extensively used as a prognostic and predictive marker for cancer diagnosis and treatment (7). Ki-67 has also been recognized as a potential prognostic biomarker in endometrial carcinoma (8-10), and is increasingly used in presurgical studies of endometrial cancer as a primary outcome measure; however, unlike its use in breast cancer, there are no guidelines for standardizing its measurement and the clinical relevance of $\mathrm{Ki}-67$ as a biomarker in endometrial cancer remains undetermined (11).

Our previous study on estrogen receptor (ER)-transfected endometrial cancer cells suggested that the stimulatory effect of estrogen on cell proliferation is exerted through the increased expression of cyclin D1 and cyclin A (12). We have also investigated various cyclins as prognostic indicators using clinical specimens of endometrial cancer, which revealed an association of cyclin A expression with progression to malignancy and a correlation with the proliferative activity and prognostic features, including histological grade (13).

Moreover, we found that cyclin A and p53 are both expressed in cells obtained from patients with endometrial carcinoma at more advanced clinical stages, using 
liquid-based cytology (14). In other studies, the expression of cyclins D1 and E was significantly correlated with the histological grade of endometrial cancer, but not with other clinicopathological parameters $(15,16)$. Among cyclins, cyclin A is most strongly associated with DNA replication, as the complex of cyclin A with CDK2 induces the G1/S transition (17). Accordingly, we selected cyclin A as a proliferation marker candidate.

Notably, several other studies have also addressed the clinical significance of elevated cyclin A expression in endometrial carcinoma (18). For example, cyclin A has been identified as an independent prognostic factor in endometrial endometrioid adenocarcinoma, and its expression correlates with the cancer grade and, to a lesser degree, with the International Federation of Gynecology and Obstetrics (FIGO) stage (19). Additionally, a multivariate analysis showed that high expression of cyclin $\mathrm{A}$ is linked to poor prognosis for patients at advanced stages of endometrial cancer, indicating the suitability of cyclin A expression as a prognostic factor for endometrial cancer (20).

Cyclin A overexpression has been reported in several other types of cancer, demonstrating prognostic value (i.e., a poor prognosis), such as in the prediction of survival or early relapse, and it has also been correlated with carcinogenesis (21-24). Furthermore, high expression of cyclin A in endometrial carcinoma has been widely linked to tumor carcinogenesis, progression, and prognosis prediction. However, a consensus has not yet been reached regarding the clinical significance of these findings.

As described above, cyclin A regulates the cell cycle and is overexpressed in cancer cells. Moreover, it has been reported as a prognostic/predictive factor in endometrial tissues (18-20). However, only a few studies have directly addressed the relationship between cyclin A and cell proliferation (25), and cyclin A has not yet been established as a proliferation marker.

Accordingly, the aim of the present study was to clarify the value of cyclin A as a marker of the cell proliferation ability in endometrioid carcinoma. For this purpose, we used two distinct types of differentiated endometrial cancer cell lines, Ishikawa and HEC-50B cells, derived from low-grade and high-grade endometrial carcinomas, respectively. We compared the expression of cyclin A with that of Ki-67 in both cell lines to clarify the usefulness of cyclin A expression as a marker of cell proliferation in endometrial cancer. Specifically, we used flow cytometry and immunocytochemical staining to compare cyclin $\mathrm{A}$ and $\mathrm{Ki}-67$ expression in the two endometrial cancer cell lines and we investigated the efficacy of cyclin A as a proliferation marker.

\section{Materials and methods}

Cell culture and cell number count. The human endometrial cancer cell lines, Ishikawa and HEC-50B, were kindly provided by Dr Kuramoto (Kitasato University, Japan) and Dr Nishida (Tsukuba University, Japan). Ishikawa cells were established from a patient with low-grade endometrial carcinoma (grade 1), and these cells express ER and progesterone receptor $(26,27)$. HEC-50B cells were established from the ascitic fluid of a patient with recurrent high-grade endometrioid carcinoma (grade 3) (28).
The cells were cultured in RPMI-1640 medium (Thermo Fisher Scientific, Inc., Waltham, MA, USA) supplemented with $10 \%$ fetal bovine serum and $1 \%$ penicillin-streptomycin (Thermo Fisher Scientific, Inc.) at $37^{\circ} \mathrm{C}$ in a humidified incubator containing $5 \% \mathrm{CO}_{2}$. At $1,2,4,6,8$ and 10 days after seeding the cultured cells, they were harvested with $0.05 \%$ trypsin- $0.02 \%$ ethylenediaminetetraacetic acid, and the cells from three dishes were counted using a Burker-Turk counter plate under an inverted phase-contrast microscope. The cell numbers were used for generating growth curves and calculating the doubling time.

Flow cytometric analysis of cyclin A and Ki-67. HEC-50B and Ishikawa cells were seeded in $60-\mathrm{mm}$ culture dishes at a density of $5 \times 10^{5}$ cells/dish, collected at various time points, and fixed using ice-cold $100 \%$ methanol. Subsequently, the expression of cyclin A was analyzed using direct-immunofluorescence flow cytometry, which was performed through double-staining with a combination of fluorescein isothiocyanate (FITC)-conjugated anti-human cyclin A2 mouse antibody (clone 11B2G3) and 7-amino-actinomycin D (7-AAD); both reagents were from Beckman Coulter (Brea, CA, USA). Ki-67 analysis was performed through single staining with an anti-mouse Ki-67 monoclonal antibody (clone PP-67, 1:100) and anti-mouse IgG/FITC (1:200); both antibodies were from Abcam (Tokyo, Japan). Cell suspensions were incubated with anti-cyclin A2 and 7-AAD for $20 \mathrm{~min}$ at room temperature $\left(20-25^{\circ} \mathrm{C}\right)$ and washed; suspensions were then incubated with anti-Ki-67 for $20 \mathrm{~min}$ at room temperature and washed. Lastly, the cells were incubated with anti-mouse IgG/FITC for $20 \mathrm{~min}$ at room temperature and then washed. The percentage of cells in each cell-cycle phase was determined through flow cytometry performed on a Cytomics FC 500 system (Beckman Coulter). In each experiment, an isotype-matched irrelevant mouse antibody was used as a negative control.

Immunocytochemical staining of cyclin A and Ki-67. Harvested cells were resuspended in phosphate-buffered saline and cytocentrifuged at 1,500 rpm for 5 min using an Auto Smear (CF-120; Sakura, Tokyo, Japan). The cells on the glass slides were immersed in $95 \%$ ethanol, and immunocytochemical staining was performed using an EnVision ${ }^{\mathrm{TM}}$ detection system (DakoCytomation A/S, Glostrup, Denmark) according to the manufacturer's recommendations. Endogenous peroxidases in the specimens were blocked with Peroxidase-Blocking Solution (DakoCytomation A/S) for $5 \mathrm{~min}$ at room temperature, and after incubation with Protein Block Serum-Free reagent (DakoCytomation $\mathrm{A} / \mathrm{S}$ ), the following primary antibodies were applied: anti-mouse Ki-67 monoclonal antibody (clone PP-67, 1:200; Abcam) and anti-mouse cyclin A2 monoclonal antibody (clone 6E6, 1:15; Abcam). Following incubation for $90 \mathrm{~min}$ at room temperature and washing, the cells on the slides were incubated with EnVision ${ }^{\mathrm{TM}} / \mathrm{HRP}$ Rabbit/Mouse secondary antibodies (DakoCytomation A/S) for $30 \mathrm{~min}$ at room temperature, and then with the chromogen, 3,3'-diaminobenzidine. Nuclei were counterstained using Mayer's hematoxylin (Wako Pure Chemical Industries, Ltd., Osaka, Japan). The specimens were examined and photographed at x200 magnification using a digital microscope 
A

\section{- HEC50B}

口Ishikawa

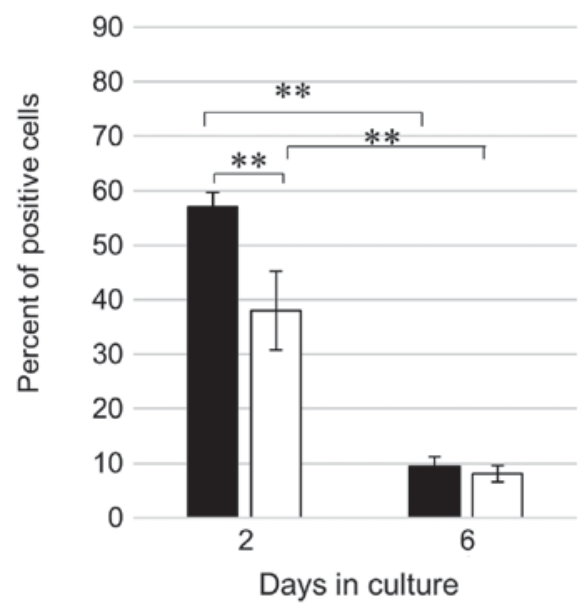

C $\square$ HEC50B

口Ishikawa

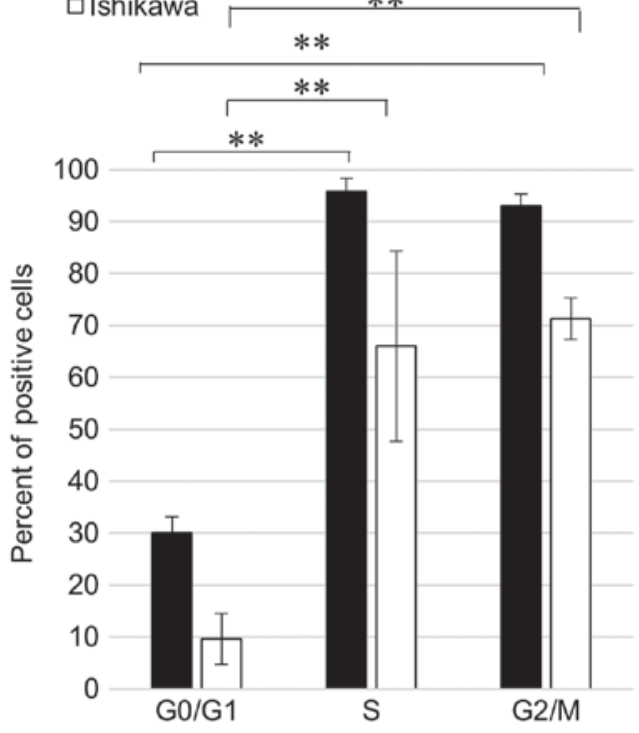

Cell cycle phase in culture of day 2

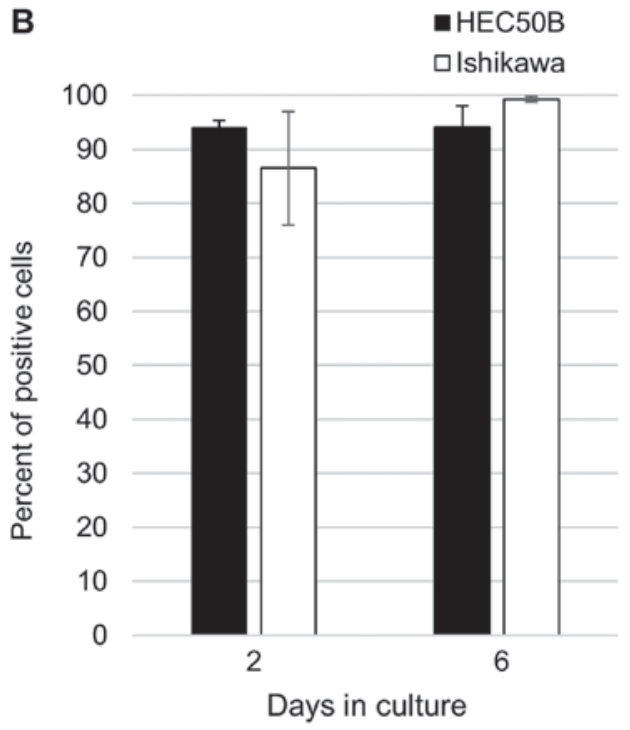

D

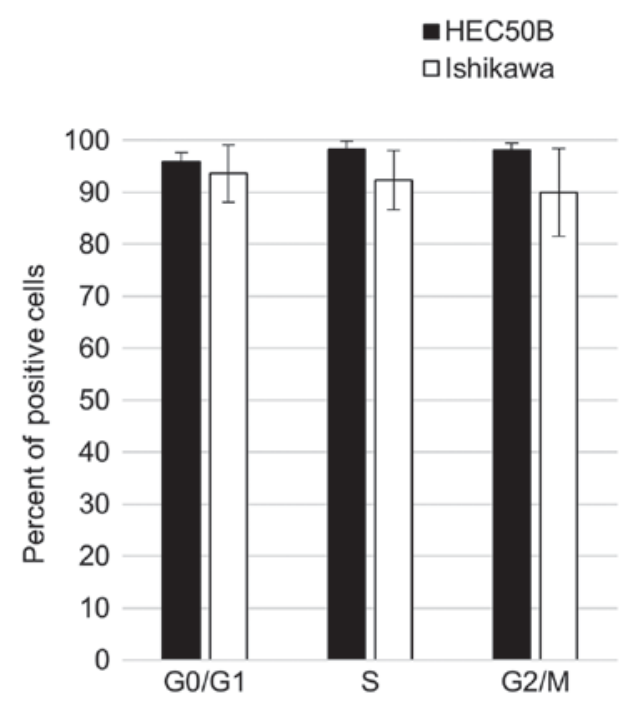

Cell cycle phase in culture of day 2

Figure 1. Flow cytometric analysis of cyclin A and Ki-67 expression. Expression of (A) cyclin A and (B) Ki-67 on days 2 and 6 of culture. Expression of (C) cyclin A and (D) Ki-67 according to cell cycle phase on day 2. HEC-50B cells, black bars; Ishikawa cells, open bars. Data are presented as mean percentages of positive cells \pm standard deviation; ${ }^{* *} \mathrm{P}<0.01$ (Tukey post hoc test).

camera (Olympus AX80 DP21; Olympus, Tokyo, Japan) interfaced with a computer. Cyclin A and Ki-67 expression was evaluated using the nuclear labeling index (\%), recorded as the percentage of positively stained nuclei in 100 cells in the hot spot.

Double immunofluorescence staining of cyclin A and Ki-67. Specimens were prepared according to the methodology given in the immunocytochemical staining section above. The primary antibodies (anti-mouse cyclin A monoclonal antibody (clone 6E6, 1:15; Abcam) and anti-rabbit Ki-67 polyclonal antibody (1:100; Abcam) were applied, incubated for $90 \mathrm{~min}$ at room temperature, and washed. Then, the cells on the slides were incubated with FITC-labeled anti-mouse $\mathrm{IgG}$ and tetramethylrhodamine (TRITC)-labeled anti-rabbit IgG
(1:40; Abcam) for $30 \mathrm{~min}$ at room temperature. Cell nuclei were counterstained with 4',6-diamidino-2-phenylindole (DAPI). Immediately after treatment, the cells were observed under a fluorescence microscope (Bz-x700; Keyence Corporation, Tokyo, Japan).

Statistical analysis. All statistical tests were conducted using BellCurve for Excel ver. 2.15 software (Social Survey Research Information, Tokyo, Japan). For multiple comparison of more than three experimental groups ANOVA analysis with the Tukey post hoc test were performed (Figs. 1 and 2), while t-tests were used for comparisons between two groups (Fig. 3). $\mathrm{P}<0.05$ was considered to indicate statistical significance. Data are presented as mean percentages of positive cells \pm standard deviation. 
A
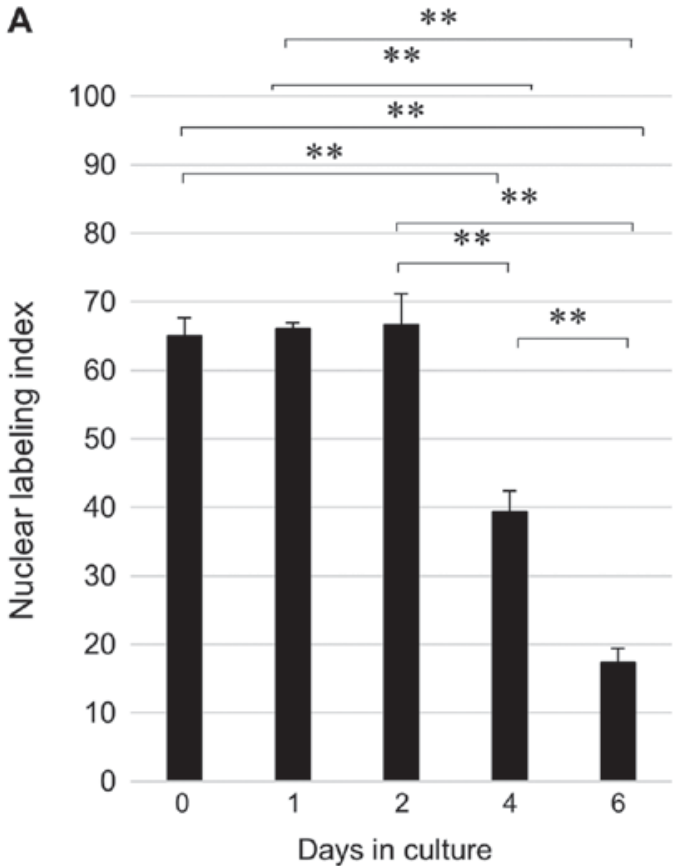

C

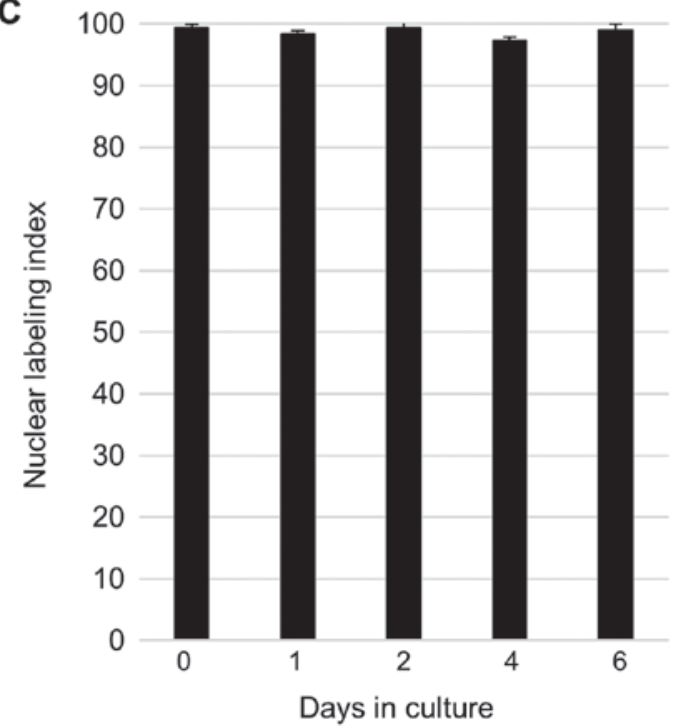

B
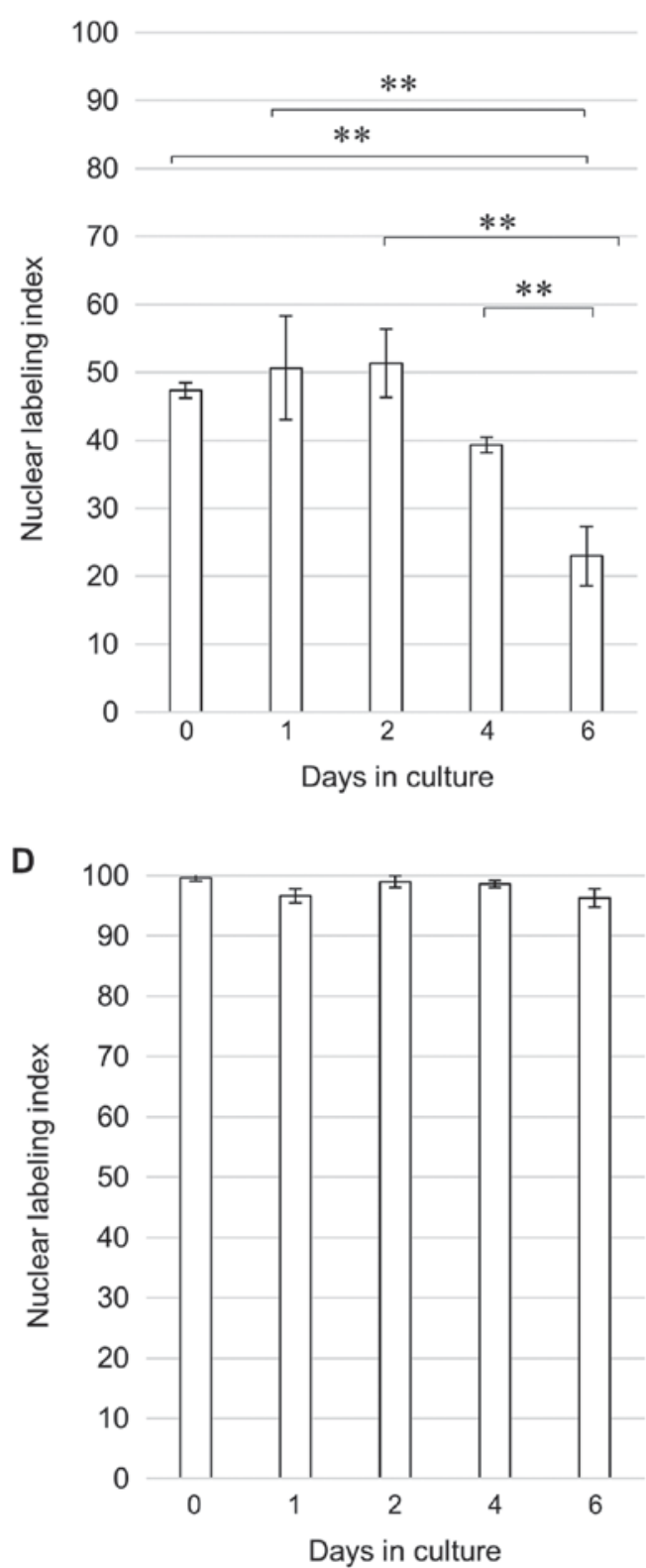

Figure 2. Time course of cyclin A and Ki-67 expression in endometrial carcinoma cell lines. Nuclear labeling index of (A) cyclin A in HEC-50B cells, (B) cyclin A in Ishikawa cells, (C) Ki-67 in HEC-50B cells, and (D) Ki-67 in Ishikawa cells. Data shown are means of nuclear labeling index \pm standard deviation; ${ }^{* *} \mathrm{P}<0.01$ (Tukey post hoc test).

\section{Results}

Cell growth curves. To validate the difference in proliferative ability between the two cell lines, we first constructed cell growth curves for HEC-50B (Fig. 4A) and Ishikawa cells (Fig. 4B). The log-phase growth of HEC-50B cells lasted for approximately four days (from day 0 to day 4 ) and was followed by a stationary phase. In contrast, the lag phase of the Ishikawa cells lasted for one day, and the log phase lasted for three days (from day 1 to day 4); the cell number then continued to increase until day 6 , after which it decreased. Calculation of the doubling time from the log phase in the growth curve revealed that the doubling time was shorter for HEC-50B cells (25.2 h) than for Ishikawa cells $(29.9 \mathrm{~h})$, thereby confirming the greater proliferative ability of the high-grade cell line.
Flow cytometric analysis of the cell cycle. For cell cycle analysis, the cellular DNA content was monitored by performing flow cytometry on cells stained with 7-AAD (Fig. 5). The cellular DNA content frequency histograms revealed the cell distribution in the three major phases of the cell cycle (G0/G1, S and G2/M). Both cell lines exhibited a reduction in the percentage of cells in the $\mathrm{S}$ and $\mathrm{G} 2 / \mathrm{M}$ phases and an increase in that in the G0/G1 phase as the culture time increased.

Expression of cyclin A and Ki-67 evaluated using flow cytometry. The flow cytometry results confirmed the expression of cyclin A and $\mathrm{Ki}-67$ in both HEC-50B and Ishikawa cells. For this analysis, the cells were collected on the second (logarithmic growth phase) and sixth day (stationary phase) 
A a-1

Day 2

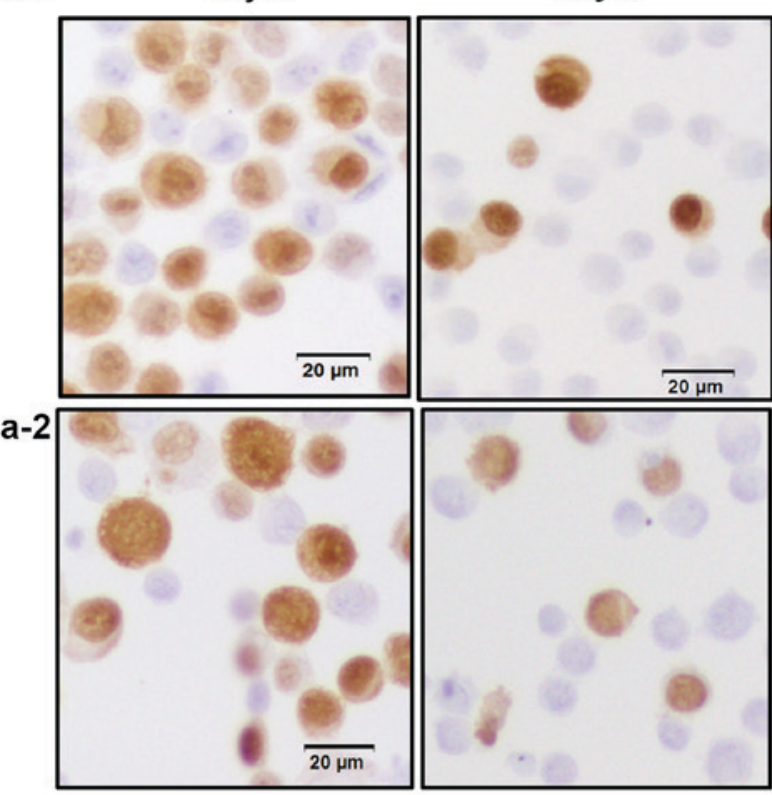

B b-1

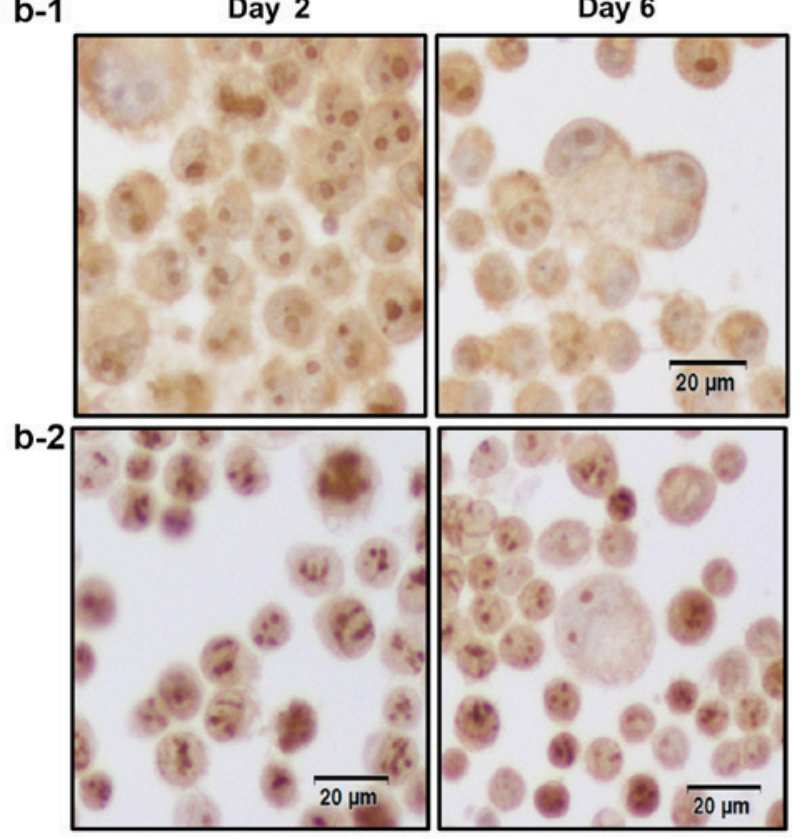

C

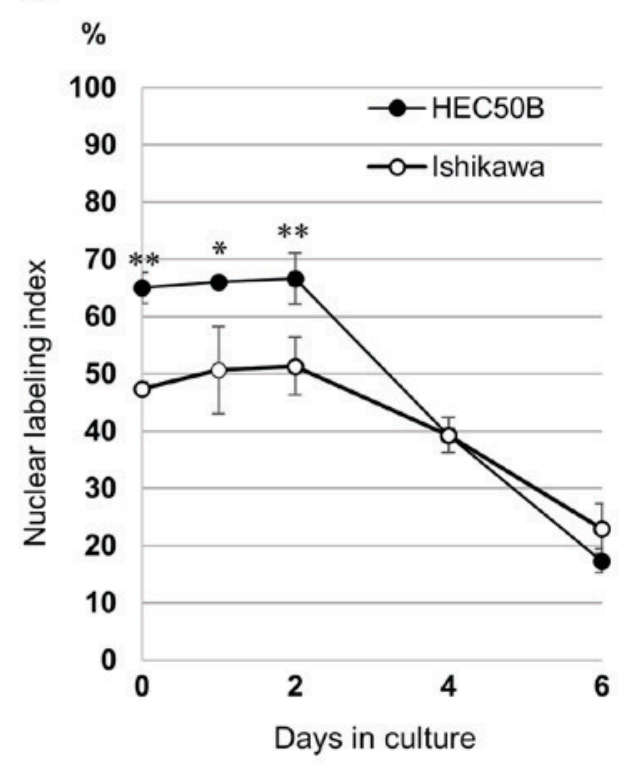

D

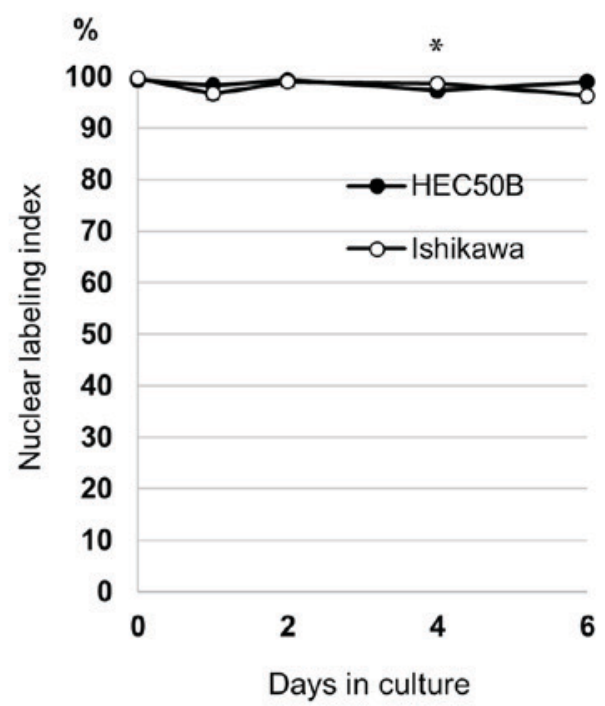

Figure 3. Time course of cyclin A and Ki-67 expression in endometrial carcinoma cell lines. Immunocytochemical staining of (A) cyclin A and (B) Ki-67. Nuclear labeling index of (C) cyclin A and (D) Ki-67. a-1 and b-1, HEC-50B cells; a-2 and b-2, Ishikawa cells. Image magnification in immunocytochemical staining: x200 magnification. Data shown are means of nuclear labeling index \pm standard deviation; ${ }^{*} \mathrm{P}<0.05,{ }^{* *} \mathrm{P}<0.01$ ( $\mathrm{t}$ test).

of culture. Cyclin A expression was significantly higher in HEC-50B cells than in Ishikawa cells on day $2(\mathrm{P}=0.0017)$ and was consistently higher on day 2 than on day 6 in both cell lines $(\mathrm{P}<0.001$; Fig. 1A). Conversely, no remarkable differences in the expression of $\mathrm{Ki}-67$ were observed between Hec50B and Ishikawa cells on day 2 and day 6 (Fig. 1B). Examination of cyclin A and Ki-67 expression during the cell cycle on day 2 revealed that, in both HEC-50B and Ishikawa cells, cyclin A expression was significantly higher in $\mathrm{S}$ and $\mathrm{G} 2 / \mathrm{M}$ phases than in $\mathrm{G} 0 / \mathrm{G} 1$ phase $(\mathrm{P}<0.001$; Fig. $1 \mathrm{C})$. In contrast, Ki-67 was expressed by roughly $90 \%$ of both the HEC-50B and Ishikawa cells and showed no notable difference in expression in the different phases of the cell cycle (Fig. 1D).
Expression of cyclin A and Ki-67 evaluated using immunocytochemical staining. Immunocytochemical staining confirmed the expression of cyclin A (Fig. 3A) and Ki-67 (Fig. 3B) in HEC-50B and Ishikawa cells. Both proteins were expressed mainly in the nucleus, although they were also partially detected in the cytoplasm in the two cell lines. Determination of the nuclear labeling index of cyclin A (Fig. 3C) revealed that cyclin A expression was higher in HEC-50B cells than in Ishikawa cells from day 0 until day 2 and showed no significant difference after day 4. Conversely, the nuclear labeling index for Ki-67 was nearly 100\%, and Ki-67 expression showed no notable difference between HEC-50B and Ishikawa cells (Fig. 3D). Comparison of the time courses of cyclin A and Ki-67 

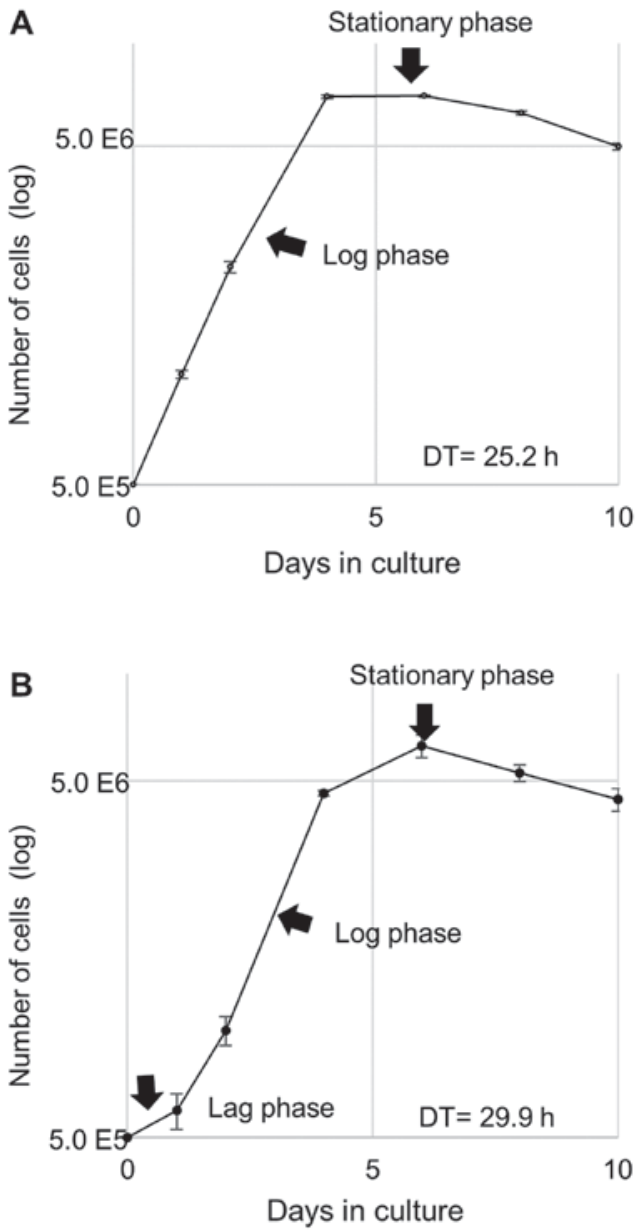

Figure 4. HEC-50B and Ishikawa cell growth curves. Growth curves of (A) HEC-50B and (B) Ishikawa cells. All measurements were performed in triplicate. HEC-50B cells grow faster than Ishikawa cells, and thus, their doubling time (DT) is shorter. $y$-axes, log of the cell number. $x$-axes, culture period of cell lines.

expression in HEC-50B and Ishikawa cells (Fig. 2) indicated that cyclin A expression was significantly higher on days 0-4 than on day 6 in both cell lines $(\mathrm{P}<0.001)$. Furthermore, we performed double immunofluorescence staining of cyclin $\mathrm{A}$ and $\mathrm{Ki}-67$ to show different expression patterns between these two proteins (Fig. 6). Both cyclin A and Ki-67 were expressed in the nuclei of endometrial carcinoma cells; where nuclear cyclin A expression was observed, the expression of $\mathrm{Ki}-67$ was also found.

\section{Discussion}

In this study, we used flow cytometry to analyze the cell cycle in two endometrial cancer cell lines featuring distinct types of differentiation. Indeed, compared to that of Ishikawa cells, HEC-50B cells had a shorter doubling time and higher proliferation rate, which is consistent with the fact that HEC-50B and Ishikawa cells were derived from grade 3 and grade 1 tumors, respectively.

Flow cytometric analysis of the cell cycle showed that both cell lines exhibited a reduction in S and G2/M phases and had a longer G0/G1 phase with prolongation of culture. These results indicated that cell proliferation ceased during culture, and that both cell lines shifted from the log phase to the
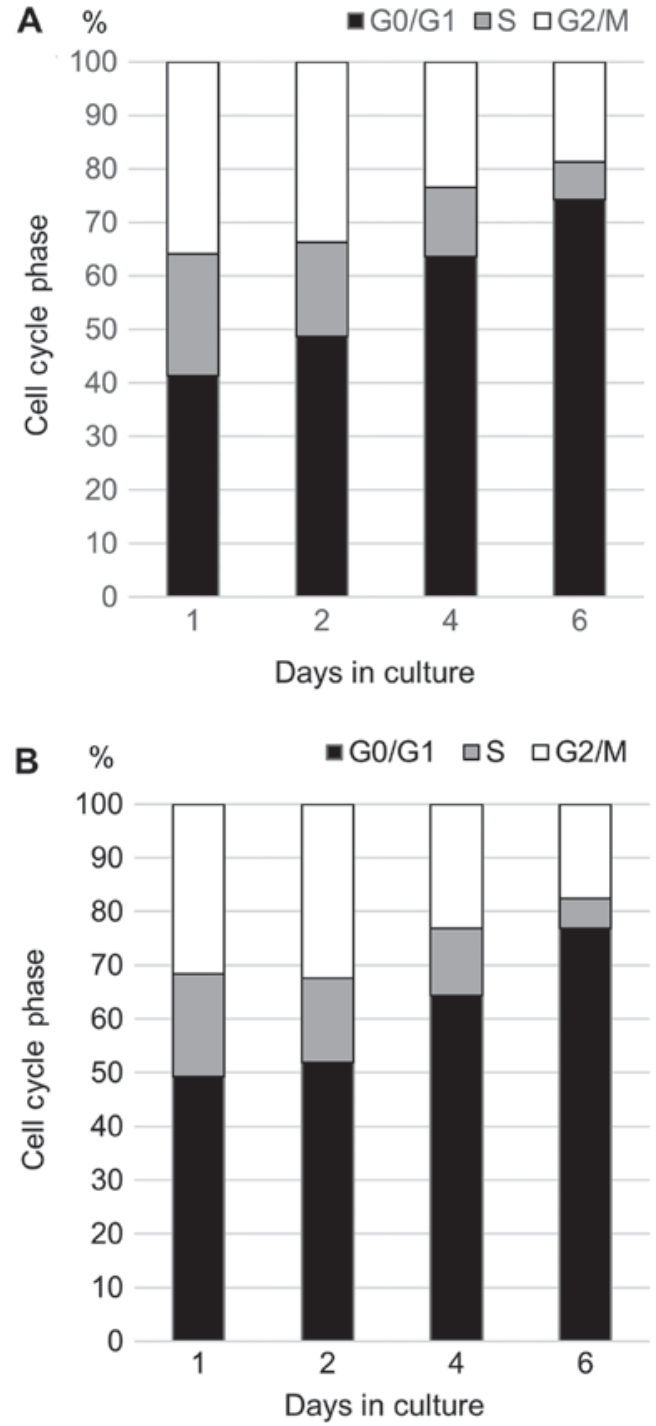

Figure 5. Analysis of cell cycles of HEC-50B and Ishikawa cells. Cell-cycle progression of (A) HEC-50B cells and (B) Ishikawa cells. G0 and G1 phases, black bars; S phase, gray bars; G2 and M phases, open bars. $y$-axes, cell distribution in the cell cycle phases. $\mathrm{x}$-axes, culture period of cell lines.

stationary phase. The S-phase fraction of cells is considered to be the strongest predictor of survival, provided that the flow cytometric methods have been carefully standardized (29). Thus, our results confirmed that high cyclin A expression in the S-phase was correlated with the high proliferation ability of endometrial cancer cell lines.

Furthermore, both flow cytometric analysis and immunocytochemical staining revealed that cyclin A expression was markedly higher in HEC-50B cells than in Ishikawa cells on day 2 of culture. Cyclin A expression was also significantly higher in both cell lines in S and G2/M phases than in G0/G1 phase. This confirms previous studies showing that cyclin $\mathrm{A}$ is specifically expressed after the $\mathrm{S}$ phase in endometrial carcinoma cells $(18,21)$.

In contrast, Ki-67 was expressed by roughly $90 \%$ of both HEC-50B and Ishikawa cells and showed no notable difference in expression in distinct phases of the cell cycle, which is also consistent with previous reports indicating that $\mathrm{Ki}-67$ expression can be observed in all phases of the cell cycle, except G0 $(5,6)$. 
A

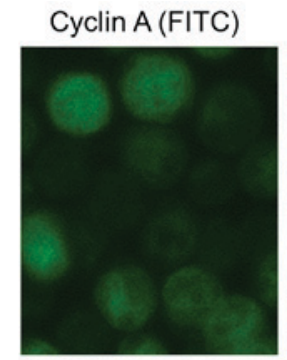

B
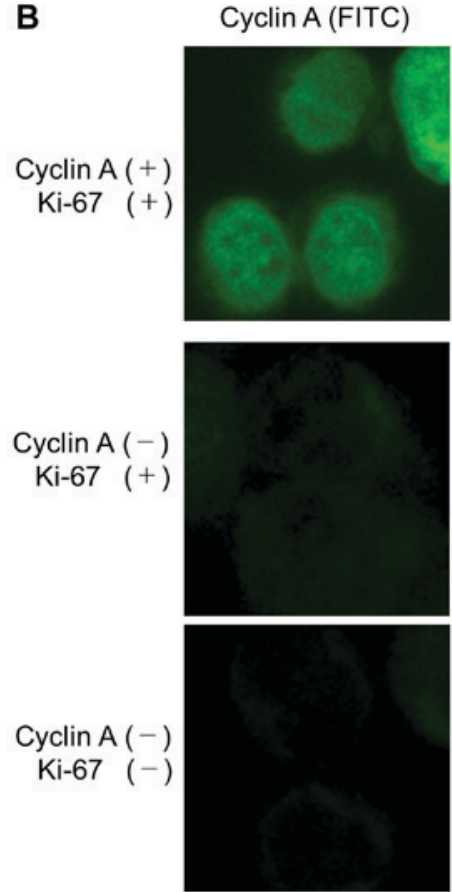

Ki-67 (TRITC)

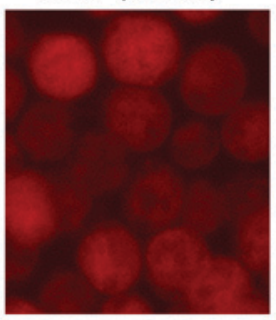

Ki-67 (TRITC)
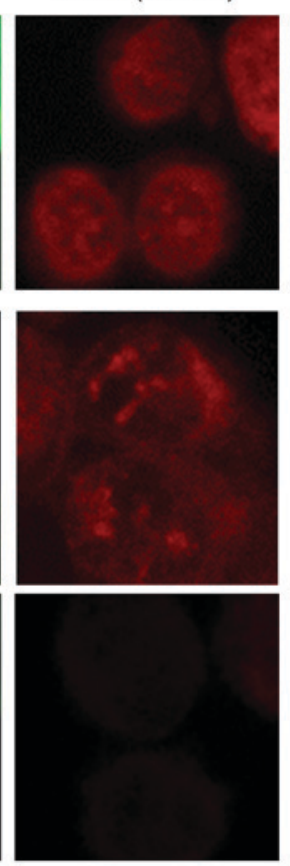

DAPI

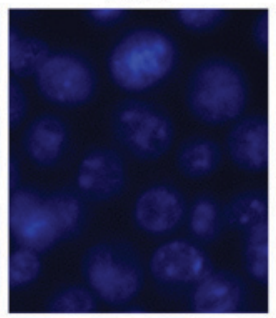

DAPI
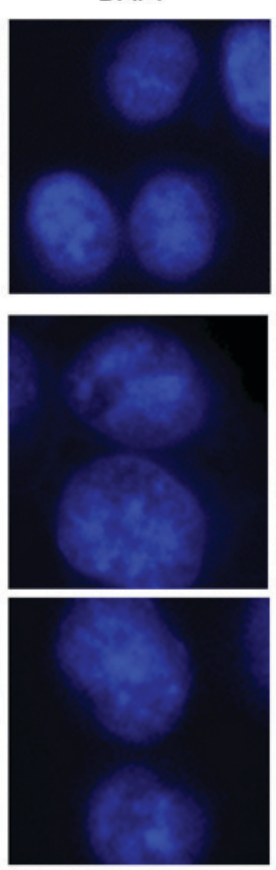
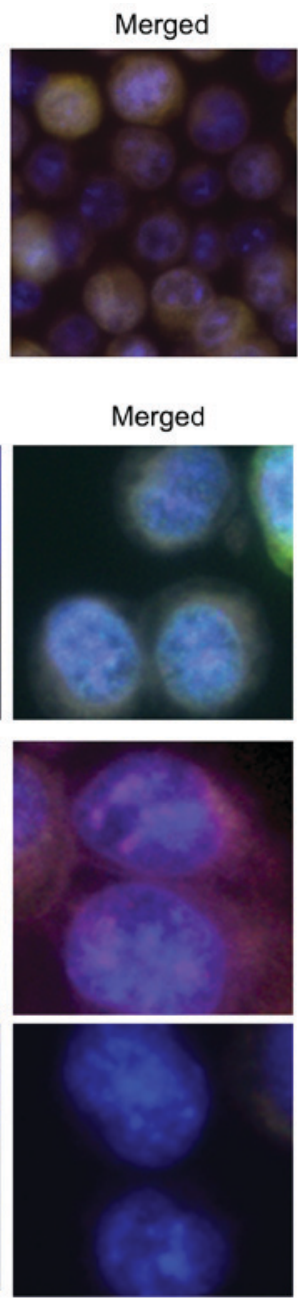

Figure 6. Double immunofluorescence staining of Ishikawa cells on day 2. (A) Representative images of double immunofluorescence staining for cyclin A and Ki-67, magnification $x 400$. The cells were stained for cyclin A (green, FITC) and Ki-67 (red, TRITC), and counterstained with DAPI (blue) to visualize the nuclei. (B) Evaluation criteria of immunofluorescence. In merged images (enlarged for clarity), cells positive for both cyclin A and Ki-67 have white nuclei, whereas cyclin A-negative, Ki-67-positive cells and double negative cells have violet and blue nuclei, respectively.

Therefore, cyclin A expression is markedly elevated in cells exhibiting increased proliferative ability and it was consistently higher on day 2 than on day 6 of culture, whereas there was no such pattern for Ki-67 expression. In fact, Ki-67 expression was higher in Ishikawa cells than in HEC-50B cells on day 6 . This is because Ishikawa cells proliferate more slowly than HEC-50B cells, and thus continued to grow for longer periods. The immunocytochemical staining confirmed the flow cytometry results, showing cyclin A expression that was higher on days 0-2 than on day 6 in both cell lines, while $\mathrm{Ki}-67$ expression did not differ markedly over time. This is because the cells enter the stationary phase and cell growth declines after four days of culture. Thus, although Ki-67 is widely recognized as a useful cell proliferation marker and prognostic factor in endometrial carcinoma (8-10), our results showed no association of Ki-67 expression with changes in the cell cycle or established variations in differentiation between the cell lines. These differences confirm that cyclin A expression is more relevant than $\mathrm{Ki}-67$ expression in endometrial carcinoma cells for detecting cells with increased proliferative ability. Furthermore, in double immunofluorescence staining, many Ki-67-expressing cells were observed and a subset of these was cyclin A positive. There is a strong possibility that cyclin A has proliferative activity.

Several studies have addressed the usefulness of cyclin A expression in endometrial carcinoma tissues as a prognostic factor using immunohistochemical staining $(19,20)$. We showed that cyclin A expression markedly increases depending on the cellular state, and that cyclin $\mathrm{A}$ is a more accurate indicator of elevated proliferation ability in endometrial cancer cell lines than Ki-67. To the best of our knowledge, this is the first study focused on cyclin A expression as a proliferation marker in this context. Thus, cyclin A expression could serve as a biomarker for the proliferation of endometrial cancer cells to provide more accurate prognostic predictions.

\section{Acknowledgements}

Not applicable.

\section{Funding}

This research was partially supported by a Hirosaki University Institutional Research Grant for Young Investigators, Research 
Funding Granted by Hirosaki University President and SCOPE of the Japan Ministry of Internal Affairs and Communications.

\section{Availability of data and materials}

The datasets used and/or analyzed during the current study are available from the corresponding author on reasonable request.

\section{Authors' contributions}

KH designed the study. HYa, KK, KT, HiY and HaY performed the experiments and analyzed the data. KH wrote the manuscript. JW contributed to analysis of the data. All authors read and approved the final manuscript.

\section{Ethics approval and consent to participate}

Not applicable.

\section{Patient consent for publication}

Not applicable.

\section{Competing interests}

The authors declare that they have no competing interests.

\section{References}

1. Yamagami W, Nagase S, Takahashi F, Ino K, Hachisuga T, Aoki D and Katabuchi H: Clinical statistics of gynecologic cancers in Japan. J Gynecol Oncol 28: e32, 2017.

2. Tran AQ and Gehrig P: Recent advances in endometrial cancer. F1000Res 6: 81, 2017.

3. ŻyłaMM,WilczyńskiJR,KostrzewaM,Księżakowska-ŁakomaK, Nowak M, Stachowiak G, Szyłło K and Stetkiewicz T: The significance of markers in the diagnosis of endometrial cancer. Prz Menopauzalny 15: 176-185, 2016.

4. Salvesen HB, Iversen OE and Akslen LA: Prognostic significance of angiogenesis and $\mathrm{Ki}-67, \mathrm{p} 53$, and p21 expression: A population-based endometrial carcinoma study. J Clin Oncol 17: 1382-1390, 1999.

5. Gerdes J, Lemke H, Baisch H, Wacker HH, Schwab U and Stein H: Cell cycle analysis of a cell proliferation-associated human nuclear antigen defined by the monoclonal antibody Ki-67. J Immunol 133: 1710-1715, 1984.

6. Schwarting R, Gerdes J, Niehus J, Jaeschke L and Stein H: Determination of the growth fraction in cell suspensions by flow cytometry using the monoclonal antibody Ki-67. J Immunol Methods 90: 65-70, 1986.

7. Li LT, Jiang G, Chen Q and Zheng JN: Ki67 is a promising molecular target in the diagnosis of cancer (review). Mol Med Rep 11: 1566-1572, 2015.

8. Kosmas K, Stamoulas M, Marouga A, Kavantzas N, Patsouris E and Athanassiadou P: Expression of Ki-67 as proliferation biomarker in imprint smears of endometrial carcinoma. Diagn Cytopathol 41: 212-217, 2013.

9. Masjeed NMA, Khandeparkar SGS, Joshi AR, Kulkarni MM and Pandya N: Immunohistochemical study of ER, PR, Ki67 and p53 in endometrial hyperplasias and endometrial carcinomas. J Clin Diagn Res 11: EC31-EC34, 2017.

10. Yang B, Shan B, Xue X, Wang H, Shan W, Ning C, Zhou Q, Chen $X$ and Luo $X$ : Predicting lymph node metastasis in endometrial cancer using serum CA125 combined with immunohistochemical markers PR and Ki67, and a comparison with other prediction models. PLoS One 11: e0155145, 2016.

11. Kitson S, Sivalingam VN, Bolton J, McVey R, Nickkho-Amiry M, Powell ME, Leary A, Nijman HW, Nout RA, Bosse T, et al: Ki-67 in endometrial cancer: Scoring optimization and prognostic relevance for window studies. Mod Pathol 30: 459-468, 2017.
12. Watanabe J, Kamata Y, Seo N, Okayasu I and Kuramoto H: Stimulatory effect of estrogen on the growth of endometrial cancer cells is regulated by cell-cycle regulators. J Steroid Biochem Mol Biol 107: 163-171, 2007.

13. Kyushima N, Watanabe J, Hata H, Jobo T, Kameya $\mathrm{T}$ and Kuramoto $\mathrm{H}$ : Expression of cyclin $\mathrm{A}$ in endometrial adenocarcinoma and its correlation with proliferative activity and clinicopathological variables. J Cancer Res Clin Oncol 128: 307-312, 2002.

14. Watanabe J, Nishimura Y, Tsunoda S, Kawaguchi M, Okayasu I and Kuramoto H: Liquid-based preparation for endometrial cytology-usefulness for predicting the prognosis of endometrial carcinoma preoperatively. Cancer 117: 254-263, 2009.

15. Kato N, Watanabe J, Jobo T, Nishimura Y, Fujisawa T, Kamata Y and Kuramoto $\mathrm{H}$ : Immunohistochemical expression of cyclin $\mathrm{E}$ in endometrial adenocarcinoma (endometrioid type) and its clinicopathological significance. J Cancer Res Clin Oncol 129: 222-226, 2003.

16. Nishimura Y, Watanabe J, Jobo T, Kato N, Fujisawa T, Kamata Y and Kuramoto H: Cyclin D1 expression in endometrioid-type endometrial adenocarcinoma is correlated with histological grade and proliferative activity, but not with prognosis. Anticancer Res 24: 2185-2191, 2004.

17. Sobczak-Thepot J, Harper F, Florentin Y, Zindy F, Brechot C and Puvion E: Localization of cyclin A at the sites of cellular DNA replication. Exp Cell Res 206: 43-48, 1993.

18. Desdouets C, Sobczak-Thépot J, Murphy M and Bréchot C: Cyclin A: Function and expression during cell proliferation. Prog Cell Cycle Res 1: 115-123, 1995.

19. Santala S, Talvensaari-Mattila A, Soini Y, Honkavuori-Toivola M and Santala M: High expression of cyclin A is associated with poor prognosis in endometrial endometrioid adenocarcinoma. Tumour Biol 35: 5395-5399, 2014.

20. Shih HC, Shiozawa T, Kato K, Imai T, Miyamoto T, Uchikawa J, Nikaido T and Konishi I: Immunohistochemical expression of cyclins, cyclin-dependent kinases, tumor-suppressor gene products, Ki-67, and sex steroid receptors in endometrial carcinoma: Positive staining for cyclin A as a poor prognostic indicator. Hum Pathol 34: 471-478, 2003.

21. Yam CH, Fung TK and Poon RY: Cyclin A in cell cycle control and cancer. Cell Mol Life Sci 59: 1317-1326, 2002.

22. Masaki T, Shiratori Y, Rengifo W, Igarashi K, Yamagata M, Kurokohchi K, Uchida N, Miyauchi Y, Yoshiji H, Watanabe S, et al: Cyclins and cyclin-dependent kinases: Comparative study of hepatocellular carcinoma versus cirrhosis. Hepatology 37: 534-543, 2003.

23. Husdal A, Bukholm G and Bukholm IR: The prognostic value and overexpression of cyclin A is correlated with gene amplification of both cyclin A and cyclin E in breast cancer patient. Cell Oncol 28: 107-116, 2006.

24. Mrena J, Wiksten JP, Kokkola A, Nordling S, Haglund C and Ristimäki A: Prognostic significance of cyclin A in gastric cancer. Int J Cancer 119: 1897-1901, 2006.

25. Paterlini P, Suberville AM, Zindy F, Melle J, Sonnier M, Marie JP, Dreyfus F and Bréchot C: Cyclin A expression in human hematological malignancies: A new marker of cell proliferation. Cancer Res 53: 235-238, 1993.

26. Nishida M, Kasahara K, Kaneko M, Iwasaki H and Hayashi K: Establishment of a new human endometrial adenocarcinoma cell line, Ishikawa cells, containing estrogen and progesterone receptors. Nihon Sanka Fujinka Gakkai Zasshi 37: 1103-1111, 1985 (In Japanese).

27. Nishida M, Kasahara K, Oki A, Satoh T, Arai Y and Kubo T: Establishment of eighteen clones of Ishikawa cells. Hum Cell 9: 109-116, 1996.

28. Kuramoto H, Hamamo M, Nishida M, Toguchi A, Jobo T, Suzuki M and Osanai K: Establishment of a cell line of human endometrial carcinoma originated from ascitic fluid. Acta Obstet Gynaecol Jpn 28: 1405-1406, 1976.

29. Nordström B, Strang P, Bergström R, Nilsson S and Tribukait B: A comparison of proliferation markers and their prognostic value for women with endometrial carcinoma. Ki-67, proliferating cell nuclear antigen, and flow cytometric S-phase fraction. Cancer 78: 1942-1951, 1996.

This work is licensed under a Creative Commons Attribution-NonCommercial-NoDerivatives 4.0 International (CC BY-NC-ND 4.0) License. 